\title{
COMPETENCIA PROFESIONAL DE FUTUROS DOCENTES, TRATAMIENTO DE LA DIVERSIDAD Y ALUMNADO INMIGRANTE
}

\section{PROFESSIONAL TEACHING COMPETENCE OF THE FUTURE TEACHERS, TREATMENT OF DIVERSITY AND INMIGRANT STUDENTS}

https://dx.doi.org/10.15304/ie.29.6269

\author{
Nuria Abal Alonso \\ nuria.abal@usc.es \\ Eduardo José Fuentes Abeledo \\ eduardo.fuentes@usc.es \\ Pablo César Muñoz Carril \\ pablocesar.munoz@usc.es \\ Universidade de Santiago de Compostela
}

\section{RESUMEN}

La investigación en la que se enmarca la aportación de este artículo responde a la recomendación del Consejo de la Unión Europea (2014) sobre la realización de estudios acerca del desarrollo de las competencias de los maestros. Nuestro objetivo es ofrecer una radiografía del nivel de competencia en las diferentes funciones y tareas docentes de los futuros profesores al final de sus estudios de Grado de Educación Infantil desde la propia perspectiva de los maestros en formación de dos Universidades públicas de Galicia. En este trabajo nos centramos en algunas de las competencias necesarias para afrontar retos educativos que se derivan de fenómenos como el multiculturalismo, el plurilingüismo, la inmigración y la diversidad. Los resultados nos alertan de la distancia que existe entre la autopercepción de competencia en las variables seleccionadas y las recomendaciones de la Comisión Europea (2019) relacionadas con las mismas.

Palabras clave: Competencias, Formación Inicial del Profesorado, Educación Infantil, Atención a la diversidad, Inmigración, Multiculturalismo.

\section{ABSTRACT}

The research that frames the contribution of this article responds to the recommendation of the Council of the European Union (2014) on conducting studies on the development of teacher competences. Our goal is to offer a snapshot of the level of competence in the different teaching functions and tasks of the future teachers at the end of their studies of the Childhood Education Degree, from the perspective of the pre-service teachers of two public Universities of Galicia. In this article, we focus on some of the necessary competences to face educational challenges that arise from phenomena such as

Recibido: 13/IX/2019. Aceptado: XI/2019 
multiculturalism, multilingualism, immigration and diversity. The results warn us of the existing gap between the self-perception of competence in the selected variables and the recommendations of the Council of Europe (2019) related to them.

Keywords: Competences, Initial teacher training, Childhood education, Attention to diversity, Immigration, Multiculturalism.

\section{INTRODUCCIÓN}

Aludir en el contexto actual en las "sociedades de la abundancia" (Lessenich, 2019) a la pluralidad de culturas, etnias y religiones, a la educación intercultural, a la acogida y cuidado de todos y de todas, también de los inmigrantes, suele generar debates, en no pocas ocasiones encarnizados, con posiciones duramente enfrentadas. En todo caso, hemos de asumir que la sociedad actual es compleja y que la escuela refleja esa complejidad, también en España. En nuestro país la variedad de culturas y de lenguas de origen de los estudiantes es una realidad derivada en gran medida de la presencia de población inmigrante que llega al país de acogida en condiciones diversas; en algunos casos se trata de familias con vidas truncadas, de adultos y niños que huyen de la pobreza, la guerra, la persecución y de la ausencia de los derechos más básicos. El caso de muchos refugiados que “escapan, corren, se lanzan al mar, se esconden, todo por no morir", como escribe Francesco Sciacca (ACNUR, 2019, p. 3), director general del Comité español de ACNUR, desvela tremendas injusticias como las que sufren millones de personas en el mundo según datos del último Informe Anual 2018 de la Agencia de la ONU para los refugiados en el que se recogen las palabras citadas.

Una escuela que asuma los principios de igualdad y equidad para ofrecer una respuesta adecuada a la diversidad y, más en concreto, a los estudiantes procedentes de otros países desde la perspectiva de acercarse al otro respetando su identidad y diferencia, apuesta por el impulso de relaciones amables, de amistad, fraternidad, solidaridad, justicia, y ha de responder al reto de ofrecer un contexto inclusivo adecuado para la convivencia armónica de todos sus miembros, con historias personales, orígenes, culturas, lenguas, capacidades, entornos socioeconómicos y conocimientos diferentes. El reconocimiento y respeto de esa diversidad, superando prejuicios, desconfianzas, creencias y prácticas erróneas, desde la defensa del derecho de equidad y calidad, y la valoración positiva de la heterogeneidad, se erige en un principio coherente con una escuela inclusiva comprometida con la educación intercultural. Principio que ha de impulsarse también en la etapa de Educación Infantil que, como reconoce la legislación española, ha de potenciar la educación integral de todos los niños y niñas.

En el momento presente, y en relación con los inmigrantes, puede resultar más difícultoso hacer realidad estas pretensiones por el impulso que están tomando acciones y discursos xenófobos alimentados por demagogos apoyándose, por ejemplo, en el ambiente caldeado por acciones terroristas del islam radical. El miedo es mal consejero y dispara la construcción de muros para excluir al "otro". Muros, fronteras físicas y administrativas, pero también, como subrayan AguilarIdáñez y Buraschi (2017), “fronteras morales, líneas de división invisibles pero extremadamente eficaces porque alimentan el rechazo y la indiferencia" (p. 106). Como declaraba Antonio Guterres, Secretario General de las Naciones Unidas el 10 de Diciembre de 2018: "Hoy vemos un crecimiento 
de las corrientes autoritarias, de intolerancia, xenofobia y racismo" (palabras pronunciadas en diciembre de 2018 durante el acto de celebración del 70 aniversario de la Declaración de Derechos Humanos). La escuela ha de ocupar un papel central para promover la defensa de valores y principios que no fomenten el crecimiento de dichas fronteras y apostar por estrategias y medidas que favorezcan la justicia y la solidaridad.

El Consejo de la Unión Europea (2014), consciente de la importancia crucial del profesorado para la mejora de la educación, ha adoptado acuerdos sobre su formación, entre ellos que la formación inicial ha de proporcionar las competencias básicas necesarias para una educación de calidad. Los docentes han de ser capaces de adaptarse a aulas multiculturales y desarrollar procesos de autorreflexión y de trabajo colaborativo, además de ser competentes para asumir roles de liderazgo. Un proyecto de escuela como el perfilado anteriormente sólo es posible con un profesorado concienciado y preparado para llevarlo a la práctica cotidiana en las aulas y en los centros, con maestros y maestras de Educación Infantil que no subestimen el bagaje cultural y lingüístico de los niños de familias inmigrantes y sepan apoyar su desarrollo en todas las dimensiones en las aulas regulares, y coordinarse y trabajar colaborativamente con otros docentes, otros profesionales y agentes de la comunidad para lograrlo. Docentes que han de adquirir conocimientos y un nivel de competencia adecuado en su formación inicial para poder seguir creciendo profesionalmente y construir una identidad profesional que responda a supuestos como los que hemos comentado, sin olvidar la relevancia de las concepciones sobre los procesos de inclusión educativa (López, Martín, Montero y Echeíta, 2013). El documento del Consejo de la Unión Europea (2014) antes citado, subraya también la necesidad de preparar a los docentes para que desarrollen formas efectivas de apoyar a diversos grupos de estudiantes, también a aquellos con necesidades especiales y/o de entornos desfavorecidos.

Nuestra aportación en este trabajo se enmarca en un proyecto de investigación (no subvencionado) acorde con supuestos como los mencionados anteriormente, y que pretende profundizar en el conocimiento de los procesos y resultados de la formación inicial de maestros y maestras de Educación Infantil en el contexto español -gallego, más en concreto- tras la implantación de los cambios propiciados por la creación del Espacio Europeo de Educación Superior y la finalización de los estudios de las primeras promociones de Grado. Nos interesa especialmente explorar cuál es la perspectiva de los propios aprendices de maestros analizando qué está ocurriendo con el desarrollo de sus concepciones, conocimiento y competencias, recogiendo su voz a través de múltiples estrategias, con confluencia de enfoques cualitativos y cuantitativos. Una línea de investigación sobre formación inicial del profesorado de Educación Infantil en auge en el contexto internacional (véase por ejemplo: Lillvist, Sandberg, Sheridan y Williams, 2014). En esta ocasión nos centraremos en una de las líneas de estudio -trabajando con datos cuantitativosexponiendo resultados y conclusiones respecto a la autopercepción de nivel de competencia de futuros docentes de centros de las Universidades de Santiago y A Coruña en varios ámbitos que nos parecen fundamentales para afrontar el reto socioeducativo que conlleva la atención a la diversidad y pensando sobre todo en la presencia en las aulas de alumnado inmigrante con culturas, y en muchos casos con lenguas diferentes a las de nuestro país. 
En nuestro proyecto de investigación asumimos que el desempeño competente como profesor de Educación Infantil, no sólo depende del nivel de competencia adquirido, sino de la autoevaluación de competencia que cada docente haga de sí mismo. Sentirse seguro en relación con los conocimientos, habilidades y actitudes que se ponen en juego para afrontar las tareas y sentirse competente, como han desvelado varias investigaciones, resulta en muchas ocasiones un factor muy relevante de competencia. Como han señalado Novick, Cauce y Grove (1996) la autopercepción de competencia profesional se refiere a cómo el maestro, o futuro maestro, interpreta su capacidad de desempeño. Por otra parte, Faria (2008) nos recuerda que: "las concepciones personales de competencia parecen ser determinantes esenciales en la consecución de objetivos orientados a la maestría, de un autoconcepto positivo y de la realización mejorada" (p. 8).

Consideramos que autoevaluación, autosatisfacción o insatisfacción, sentimiento de competencia y posibilidades de mejora están íntimamente relacionados. Lo que cada futuro docente piensa sobre sí mismo desempeña un papel importante en su realización personal y profesional.

Por otra parte, nos parece imprescindible analizar las implicaciones que los resultados en relación con la autopercepción del nivel de competencia de estos futuros docentes han de tener en diversos ámbitos y agentes en los procesos formativos, tanto para los propios futuros maestros, como para los formadores y, en su conjunto, la institución que ha de formarlos y acreditarlos en relación con las competencias que se consideren adecuadas. Necesitamos formar docentes que se sientan con un mínimo de confianza en sus competencias para que en el momento en que comiencen su andadura como profesionales, se muestren abiertos a evaluarse, a reflexionar en profundidad sobre su quehacer y a seguir mejorando.

\section{OBJETIVOS}

El Informe de la Educación y la Formación de 2019 de la Comisión Europea insta nuevamente a España a avanzar en el acceso universal a la educación infantil y a poner en marcha cambios en la formación docente. En nuestra opinión, estos cambios han de tener en cuenta los resultados de investigación en cada contexto que diagnostiquen qué está sucediendo, por ejemplo, en la formación inicial de docentes. El Consejo de la Unión Europea (2014) acordó que las instituciones de educación superior llevasen a cabo investigaciones sobre el desarrollo de las competencias de los maestros. Nuestro estudio responde a esta recomendación.

El objetivo general de la investigación en la que se enmarca este trabajo, es ofrecer una radiografía del nivel de competencia en las diferentes funciones y tareas de los futuros docentes que cursan el Grado de Educación Infantil al final de sus estudios, desde la propia perspectiva de los maestros en formación de dos Universidades públicas de Galicia. También nos interesa estudiar si existen diferencias entre los futuros docentes de los tres centros formativos considerados en relación con el nivel de competencia autopercibido. En concreto, en esta aportación nos centraremos en conocer cuál es el nivel de competencia autopercibido por futuros docentes del Grado de Educación Infantil de dos universidades públicas de Galicia (Universidad de Santiago de Compostela con dos centros formativos: Facultad de Ciencias de la Educación de Santiago y de Formación del Profesorado de Lugo) y Universidad de A Coruña (Facultad de Ciencias de la Educación), para 
afrontar algunos de los retos educativos en las aulas de Educación Infantil que se derivan del fenómeno del multiculturalismo, plurilingüismo, inmigración y diversidad.

\section{METODOLOGÍA}

\subsection{Participantes}

Sobre una población de 308 estudiantes del último curso del Grado de Maestro/a en Educación Infantil, se recogieron 225 encuestas, empleándose para ello un muestreo no probabilístico por conveniencia (Cohen y Manion, 1990). La distribución de los datos poblacionales y muestrales segmentados por centro de estudios se reflejan a continuación en la tabla 1.

\begin{tabular}{|l|c|c|c|}
\hline \multirow{2}{*}{ Centro de Estudios } & \multirow{2}{*}{ Población } & Muestra de estudiantes encuestados \\
\cline { 3 - 4 } & & n & $\begin{array}{c}\text { Porcentaje respecto a la } \\
\text { población }\end{array}$ \\
\hline $\begin{array}{l}\text { Facultad de Ciencias de la Educación. } \\
\text { (Universidad de Santiago). }\end{array}$ & 104 & 73 & $70.19 \%$ \\
\hline $\begin{array}{l}\text { Facultad de Formación del Profesorado } \\
\text { (Universidad de Santiago). }\end{array}$ & 81 & 57 & $70,37 \%$ \\
\hline $\begin{array}{l}\text { Facultad de Ciencias de la Educación } \\
\text { (Universidad de A Coruña). }\end{array}$ & 123 & 95 & $77,23 \%$ \\
\hline TOTALES & $\mathbf{3 0 8}$ & $\mathbf{2 2 5}$ & $\mathbf{7 3 , 0 5 \%}$ \\
\hline
\end{tabular}

Tabla 1. Datos poblacionales y muestrales. Fuente: Elaboración propia.

Cabe señalar en relación con el género que la inmensa mayoría de encuestadas son mujeres (93\%) frente a un escaso $7 \%$ de hombres. Subrayemos también que el 60,4\% de la muestra se sitúa entre los 21 y 23 años de edad, el 28\% entre los 24 y 26 años, el 5,8\% entre los 27 y 29 años, el mismo porcentaje que alcanzan los que tiene más de 30 años.

\subsection{Procedimiento}

Se llevó a cabo la aplicación de un extenso cuestionario al alumnado de la titulación del Grado de Educación Infantil en dos Facultades (de Ciencias de la Educación y Formación del Profesorado) de Universidad de Santiago de Compostela y en la Facultad de Ciencias de la Educación de la Universidad de A Coruña. La aplicación del instrumento contó con la presencia y control de los investigadores y la colaboración previa de profesorado de los tres centros citados. El proceso se llevó a cabo bien al inicio o al final de una de las sesiones de trabajo de materias de $4^{\circ}$ que estaban cursando al final del segundo cuatrimestre del último curso de la titulación, siendo respondido el cuestionario por aquellos futuros docentes que habían realizado sus períodos de Practicum, asistían regularmente a clase y se encontraban al término de sus estudios. Los estudiantes colaboraron de forma voluntaria, incluso con entusiasmo, al presentarles un instrumento que invitaba a la reflexión sobre su propio nivel de competencia profesional. 


\subsection{Instrumento y variables seleccionadas}

El instrumento de recogida de información ha sido un cuestionario que incorpora un total de 107 ítems y que ha sido construido específicamente para la investigación en la que se enmarca esta aportación ("Cuestionario sobre el desarrollo de competencias docentes en la formación inicial del profesorado de Educación Infantil"). Consta de 12 ítems referidos a datos generales (de contextualización e identificación de carácter personal y académico, aspectos sobre competencia en lengua extranjera y experiencias laborales en relación con la Educación Infantil); 86 ítems de competencias específicas para el desempeño como docente de Educación Infantil; 3 ítems de competencia global; 3 de valoración general de la formación recibida y 3 cuestiones abiertas. Es preciso señalar que se ha empleado una escala Likert para recoger datos sobre el nivel de autopercepción de competencia. Se define con un valor que va desde 1 (que el futuro docente elige cuando se siente "nada" competente) a 4 ("mucho"), con otras dos opciones entre ambos valores: 2 ("poco") y 3 ("bastante"). Para la selección y redacción de los ítems referidos a las diferentes competencias objeto de estudio hemos adaptado el marco conceptual en torno a cuatro ámbitos de desempeño y desarrollo profesional (docente, tutorial, institucional y personal) y varias funciones y tareas (adaptando una propuesta de Fuentes Abeledo, 2009, y considerando las funciones y tareas del profesorado establecidas en la LOE -artículo 91-), considerando varias fuentes para la selección y definición de las competencias concretas (Orden ECI/3854/2007 de 27 de Diciembre que establece los requisitos para la verificación de los títulos universitarios que habilitan para el ejercicio de la profesión de maestro en Educación Infantil; literatura curricular y propuestas de competencias de reconocido prestigio en el ámbito de la Educación Infantil y documentos curriculares de las Universidades en las que se han recogido los datos).

Respecto a la validez y fiabilidad del instrumento hemos llevado a cabo una prueba piloto con 16 alumnos (pertenecientes a los tres centros en los que se han recogido los datos y también a un centro universitario de formación de docentes de la Universidad de Vigo) y, además, en la validación de su contenido han participado diversos expertos docentes e investigadores de las Universidades de Santiago de Compostela y A Coruña. Se ha utilizado como medida de fiabilidad y de consistencia interna Alfa de Cronbach, obteniéndose un resultado de 0,962 para todo el cuestionario y de 0,730 para la dimensión a la que pertenecen las variables analizadas en el presente manuscrito. Dado que los valores a obtenidos no son en ningún caso menores de 0,70 (McMillan y Schumacher, 2010), debemos considerar que el instrumento posee un elevado nivel de fiabilidad y una alta consistencia interna. También se ha realizado un análisis de la correlación de cada ítem con el total. De acuerdo con Nurosis (1993), ésta debería ser mayor a 0,3 para aceptar que los ítems están homogéneamente relacionados con la escala a la que pertenecen. En nuestro caso, existían algunos ítems inferiores a dicha puntuación, sin embargo se decidió mantenerlos en el cuestionario ya que su eliminación no comportaba un aumento significativo a la fiabilidad de la encuesta.

Una vez aplicado el cuestionario y codificados los datos, se llevó a cabo el análisis de los mismos mediante procedimientos estadísticos utilizando la aplicación informática SPSS versión 20.

Para este trabajo hemos seleccionado tres variables del cuestionario en relación con competencias profesionales que consideramos relevantes para poder trabajar con alumnado 
inmigrante. Dos de ellas referidas al ámbito lingüístico y una referida a la integración de alumnos con dificultades. Dos se corresponden con el ámbito de la actuación en la fase interactiva de la enseñanza y una (identificar disfunciones en el lenguaje) a la evaluación, todas ellas incluidas en la dimensión "docente" según el marco conceptual al que hemos hecho referencia. Especialmente a considerar son los resultados obtenidos sobre la competencia en "Abordar con eficacia situaciones de aprendizaje de lenguas en contextos multiculturales y multilingües" (variable 43), y otra del ámbito lingüístico: "Identificar posibles disfunciones en el lenguaje" (variable 78). Por otra parte, la tercera variable considerada en esta aportación se relaciona directamente con la atención a la diversidad con carácter global: "Favorecer la integración educativa de alumnos con dificultades" (variable 46).

\section{Resultados}

Entre las recomendaciones del Consejo de la Unión Europea de 22 de Mayo de 2019 relativas a los sistemas de educación y cuidado de primera infancia de alta calidad, varias se relacionan con el aprendizaje lingüístico y con la necesidad de que los profesionales respondan a las necesidades individuales del alumnado procedentes de diferentes entornos. De las variables seleccionadas para este trabajo y que se relacionan con dichas recomendaciones, destacamos los bajos niveles de competencia percibida por los futuros docentes de Educación Infantil en dos que tienen que ver con el ámbito lingüístico y que estimamos muy relevantes para trabajar con alumnado inmigrante, sobre todo en aquellos casos en que su lengua de comunicación sea diferente a la lengua vehicular en la escuela. La Figura 1 refleja las medianas obtenidas para las variables consideradas.



Figura 1. Medianas de las variables del estudio. Fuente: elaboración propia. 
Para nuestros comentarios señalemos que en lo que denominamos "Zona Baja” englobamos los porcentajes correspondientes a los grados "nada" y "poco" de la escala, y en la "Zona Alta" los correspondientes a "bastante" y "mucho".

La variable 43 "Abordar con eficacia situaciones de aprendizaje de lenguas en contextos multiculturales y multilingües" alcanza la Media más baja $(2,23)$ de las 86 incorporadas al cuestionario, obtiene una mediana de 2 y una Desviación Típica de 0,730 . Además, como refleja la Figura 2 resulta muy significativo que en la Zona Baja el porcentaje sea del 64,9\%, mientras que en la Zona Alta sólo el 34,7\%.

\section{p43 Abordar con eficacia situaciones de aprendizaje de lenguas en contextos multiculturales y multilingües}

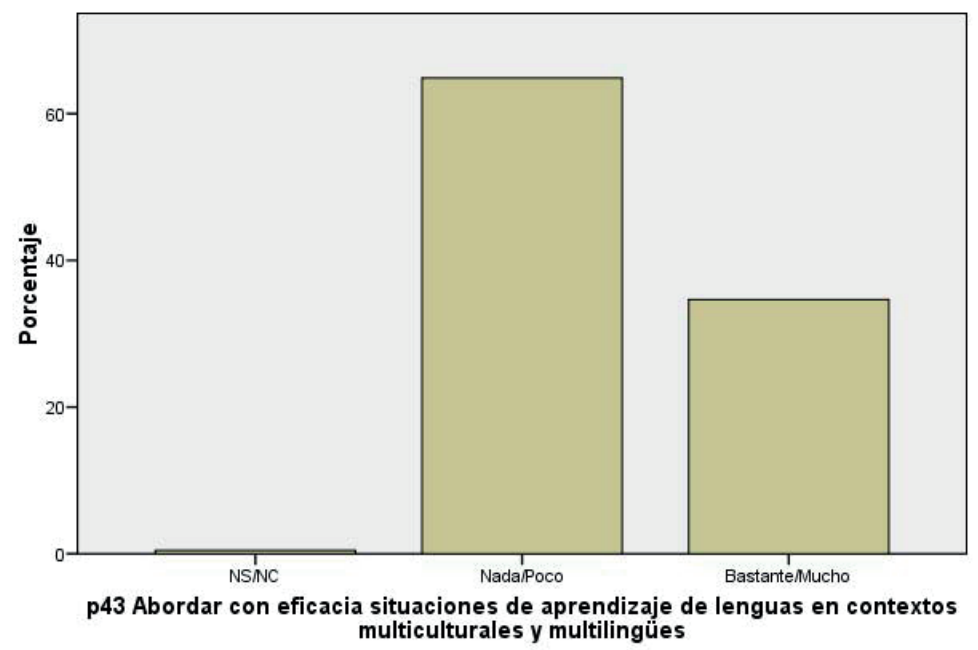

Figura 2. Porcentajes obtenidos en la variable 43 "Abordar con eficacia situaciones de aprendizaje de lenguas en contextos multiculturales y multilingües”. Fuente: elaboración propia.

Además, cabe destacar también que el 13,8\% selecciona la opción "nada" y únicamente el $8,4 \%$ la opción "mucho", repartiéndose el resto de forma bastante equilibrada (40,4\% para la opción "poco" y 46,2\% para la opción "bastante").

Diversos estudios en nuestro contexto han destacado la importancia que le da el profesorado en ejercicio al dominio de la lengua para la adecuada integración del alumnado extranjero (por ejemplo: Navarro, Huguety Sansó, 2014) y, por otra parte, investigaciones como la de Santos Rego, Godás y Lorenzo (2013) en el contexto gallego — con dos lenguas oficiales - constatan que el alumnado inmigrante tiene un nivel de fracaso escolar muy superior al de sus pares y que éste se relaciona con las diferencias en el nivel de competencia lingúística. A la luz de los datos de nuestra investigación hemos de reflexionar acerca de la inseguridad que puede sentir una parte importante de los futuros docentes de Educación Infantil para abordar situaciones de aprendizaje 
de lenguas en los contextos citados. Se desvela un panorama poco halagüeño dada la importancia de la lengua para regular y planificar la conducta en relación con uno mismo y con los otros y progresar académicamente. Los resultados en torno a esta competencia son motivo de preocupación pensando en que los futuros docentes han de estar mínimamente preparados para poder trabajar tanto dentro como fuera de una aula ordinaria con la creciente diversidad del alumnado, también socio-lingüística, y apoyar el desarrollo tanto de la competencia lingüística conversacional como la cognitivo-académica (Cummins, 2000), y procurar la integración lingüística del alumno inmigrado (Vasseur, 2006; Ibarra, 2012)

Cabe pretender que el profesorado no sólo sostenga concepciones alejadas de la idea tan extendida de la bondad de las aulas homogéneas frente a las heterogéneas, se aleje de lecturas de las dificultades del alumnado desde determinados prejuicios y estereotipos (Moreno, 2006), y mantenga una actitud positiva ante la diversidad. Hemos de ser ambiciosos y ofrecer la mejor capacitación para que estos futuros docentes se sientan más seguros y afronten la organización de contextos desarrollando una práctica educativa que tenga sentido para todos los alumnos reconociendo aquello que los estudiantes extranjeros llevan a las aulas, como por ejemplo su lengua, su identidad y sus conocimientos, como defiende Vila (2007). Para que actúen apoyando el desarrollo de la competencia lingüística de los pequeños en la escuela de Educación Infantil desde la perspectiva de favorecer la construcción de una sociedad intercultural, potenciando la implicación del conjunto de la comunidad educativa (Baráibar, 2005), y promoviendo valores propicios para aumentar la cohesión y habilidades, conocimientos y actitudes que favorezcan la inclusión social.

Entendemos que sólo de esta forma se estará en condiciones iniciales de convertir las aulas ordinarias en el lugar principal para el aprendizaje de la lengua escolar teniendo en cuenta las desventajas que pueden suponer las aulas de acogida "cerradas" tal y como denunciaba hace ya mucho tiempo Siguán (1998) y, en todo caso, siempre manteniéndose muy sensible y abierto a la cooperación ante la imprescindible coordinación de todo el profesorado que atienda al mismo alumnado, sea en aulas ordinarias o en aulas específicas de acogida, siempre desde la defensa de una escuela inclusiva que planifique adecuadamente la progresión en las necesarias habilidades conversacionales con el fin de que, en el menor tiempo posible, todo el alumnado pueda participar de forma activa en el aula ordinaria (Ribas, Iglesias, Siqués y Vila, 2006). En este sentido, las aulas de Educación Infantil constituyen un contexto enormemente propicio para fomentar la participación de todos los alumnos en los avances pertinentes propiciadores del dominio lingüístico de la lengua.

Por todo lo que venimos comentando nos preocupa también que una de las Medias más bajas en relación con las 86 competencias abordadas en el cuestionario sea la referida a "Identificar posibles disfunciones en el lenguaje". Se corresponde con la variable 78 del cuestionario, con una Media de 2,44, una mediana de 2 y DT de 0,639. Para esta variable se alcanza en Zona Baja un porcentaje superior al obtenido en la Zona Alta (56,45\% frente a 43,6\%) como se puede apreciar en la Figura 3. Además, 8 sujetos (el 3,6\%) han escogido la opción "nada", y sólo 10 sujetos (el 4,4\%), se consideran muy competentes, mientras que el 52,9\% (119 sujetos) se autoperciben como "poco" competentes y el 39,1 \% (88 sujetos), se autovaloran como "bastante competentes". 


\section{p78 Identificar posibles disfunciones en el lenguaje}

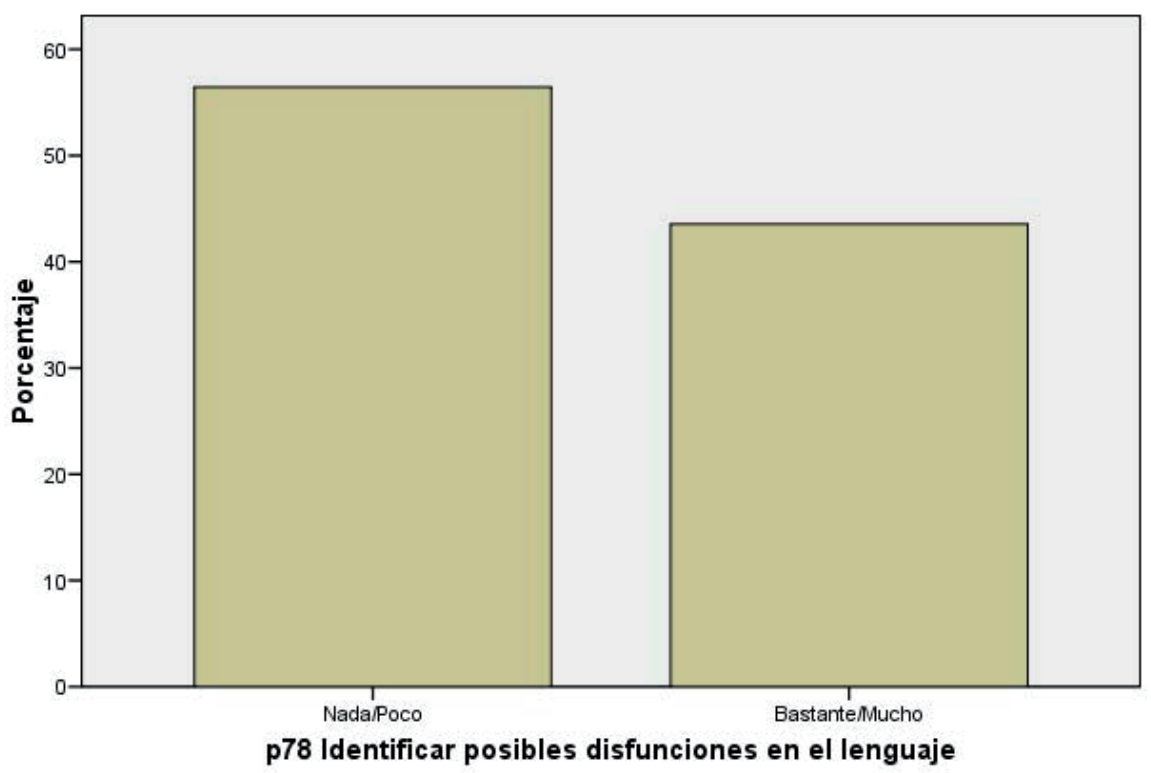

Figura 3. Porcentajes obtenidos en la variable 78 "Identificar posibles disfunciones en el lenguaje". Fuente: elaboración propia.

Nos parece imprescindible una adecuada y profunda preparación para abordar con el alumnado de Educación Infantil las disfunciones en el ámbito lingüístico. Sólo así podemos evitar una intervención poco informada y favorecedora del etiquetaje arbitrario y antieducativo que tanto daño puede provocar, sobre todo si prevalecen prejuicios y estereotipos, tan nefastos para el aprendizaje de la lengua y, desgraciadamente, aún presentes en ocasiones, también en relación con inmigrantes que aprenden una nueva lengua (Moreno, 2006, Ibarra, 2012).

La normativa educativa estatal reconoce, en general, las necesidades de apoyo del alumnado inmigrante. Un número elevado son considerados "alumnos con dificultades". Resulta de gran interés conocer cómo se sienten de competentes los futuros docentes para desenvolverse en la fase interactiva de la enseñanza. Por ello nos detenemos en presentar los datos respecto a "Favorecer la integración educativa de estudiantes con dificultades" — variable 46 del cuestionario-. En la Zona Baja se alcanza un porcentaje de 47,1\% (con un 43,1\% que eligieron la opción "poco", frente aun escaso $4 \%$ que optó por "nada") mientras que en la Zona Alta se refleja un porcentaje levemente superior (52,4\%, con un 42,2\% que eligió la opción "bastante" y un 10,2\% "mucho") siendo la Media de 2,58, la mediana de 3 y la DT de 0,747. Los porcentajes por Zonas Baja y Alta se reflejan en la Figura 4 que aparece a continuación. 


\section{p46 Favorecer la integración educativa de estudiantes con dificultades}

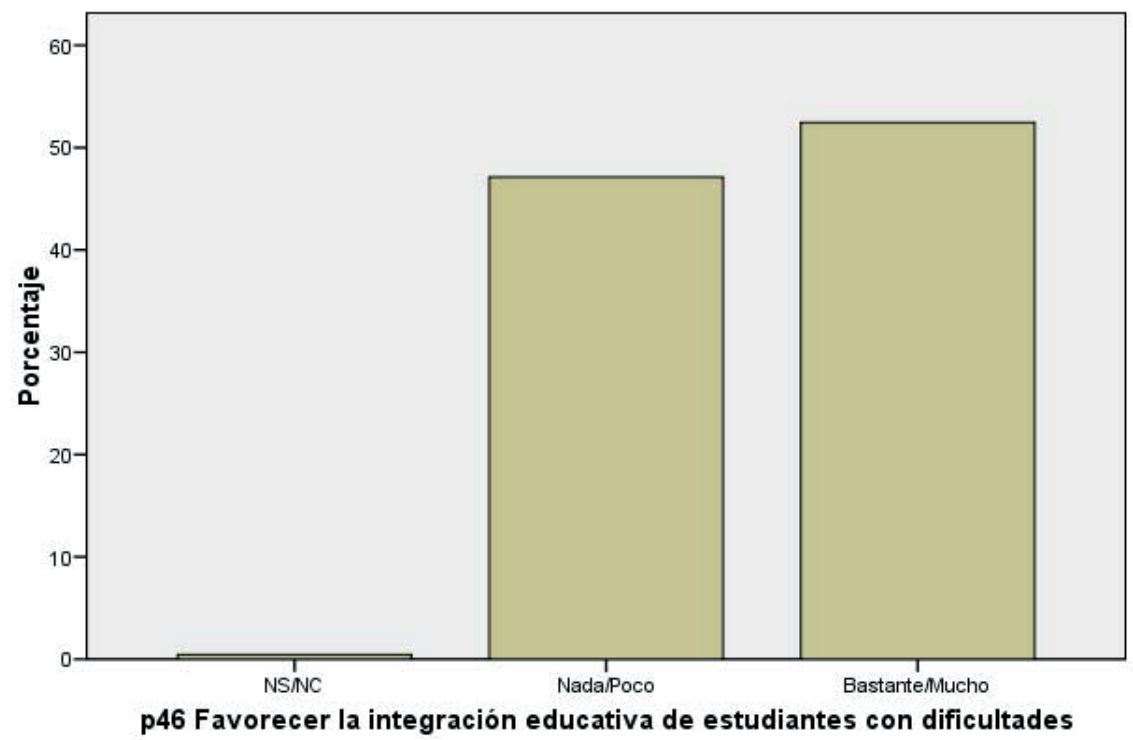

Figura 4. Porcentajes obtenidos en la variable 46 "Favorecer la integración educativa de estudiantes con dificultades". Fuente: elaboración propia.

Como hemos comentado en la introducción, una escuela que busque la igualdad y el respeto a los derechos humanos ha de hacerlo a través de una adecuada atención a la diversidad, es decir, cuando todo el alumnado perciba que sus características culturales y personales y sus vivencias son tenidas en cuenta en la vida del aula y del centro, y se apueste claramente por el rechazo a las relaciones de exclusión. Pero si bien el marco legal español se orienta en este sentido, no puede hacerse realidad en los centros y en las aulas si los docentes no saben, o no quieren, abordar el tratamiento de la diferencia de forma coherente con los principios expresados anteriormente.

Saber "favorecer la integración" requiere competencia del profesor en todas las dimensiones del desempeño y desarrollo profesional que hemos considerado en la investigación (personal, docente, institucional y tutorial).

El profesorado en ejercicio, y el futuro docente, ha de conocer y saber usar los recursos adecuados, que han de estar a disposición de forma permanente, pero también convertir en objeto de análisis, reflexión e investigación concepciones y prácticas psicopedagógicas, curriculares y organizativas (López, Martín, Montero y Echeíta, 2013), comprometiéndose en una incesante búsqueda de soluciones a los múltiples problemas que surgen en la cotidianiedad cuando se intentan desarrollar prácticas inclusivas en un contexto social complejo en que, por ejemplo, la lucha despiadada por el éxito personal se hace muy presente y puede dificultar el asentamiento de un enfoque inclusivo que pretende potenciar la convivencia y el progreso de todos y todas respondiendo al principio de la igualdad de derechos. 
En no pocas ocasiones, aunque desde el punto de vista administrativo se apueste por una escolarización inclusiva de niños "con dificultades", los excesos de un ambiente de competitividad salvaje y de individualismo exacerbado, pueden socavar la construcción de una auténtica propuesta inclusiva. Una propuesta que, entre otros elementos, ha de potenciar la comprensión de dificultades y limitaciones del otro - analizando por ejemplo, erróneas interpretaciones de determinados comportamientos, esterotipos, prejuicios...- para poder colaborar, con implicación de todos, en un adecuado desarrollo y aprendizaje individual y de grupo a través de la ayuda mutua en la vida cotidina de un aula de Educación Infantil — para abrocharse el mandilón, para construir una pared con cajas en el desarrollo de un proyecto, para encontrar el objeto perdido...-

\section{CONCLUSIONES}

Los resultados que hemos presentado nos alertan de la distancia que existe entre la autopercepción de competencia de los participantes en este estudio en las variables seleccionadas, y las recomendaciones de la Comisión Europea (2019) relacionadas con las mismas. Los datos que reflejan las valoraciones de futuros docentes de Educación Infantil poco antes de terminar su formación inicial como maestras y maestros, y realizado ya el Practicum de la titulación que constituye un eje muy relevante para el desarrollo de sus competencias profesionales, nos aportan elementos e indicadores suficientemente destacados para ser tenidos en cuenta y reflexionar sobre algunos de los contenidos, actividades, procesos y agentes intervinientes en la formación inicial más adecuados para que los futuros docentes puedan desarrollar prácticas educativas de la mayor calidad, tanto con el alumnado en general como pensando de forma especial en escolares de origen inmigrante con lenguas y culturas diferentes a las autóctonas sin dominio de la lengua vehicular habitual en la escuela, un alumnado que, subrayémoslo, suele presentar en su recorrido académico altas tasas de fracaso escolar. El bajo nivel autopercibido de competencia y, por lo tanto, el grado de inseguridad que estos futuros docentes pueden sentir para el ejercicio profesional añade un elemento de preocupación respecto a la actitud y motivación que pueden adoptar ante niños inmigrantes. Todo ello asumiendo que la autopercepción de competencia no tiene por qué coincidir necesariamente con el grado de competencia real que puede demostrarse.

Consideramos oportuno introducir mejoras en la formación para sensibilizar y preparar a los futuros docentes en la identificación de dificultades comunicativas habituales del alumnado que tiene una lengua materna diferente a la vehicular de la escuela, propiciando la reflexión sobre cuestiones didácticas importantes como los contextos y estilos de comunicación docente más adecuados, y la detección y corrección de errores para establecer prioridades en la tarea diaria (Chireac, Serrat y Huguet, 2011). No resultan halagüeños los resultados presentados respecto a las dos primeras competencias analizadas dada la importancia de la lengua (instrumento simbólico por excelencia), tanto para regular la conducta en relación con uno mismo como en el trato con el otro y lograr una adecuada progresión académica, es decir, usando los conceptos de Cummis (2000), para desarrollar la Competencia Lingüística Conversacional y la Competencia Cognitivo-Académica y, de forma más global, favorecer el espíritu democrático potenciando la competencia comunicativa (Lomas y Ruiz, 2011), respetando la diversidad y apostando por la inclusión y la educación intercultural. 
Los docentes se enfrentan a nuevos retos, entre ellos la diversidad en las aulas derivada, entre otras causas, de la presencia de niños y niñas de familias inmigrantes y han de trabajar con alumnado con culturas y lenguas diversas, y escolares con dificultades de aprendizaje. Destacamos sobre todo la necesidad de prestar mayor atención en la formación inicial a las dos competencias del ámbito lingüístico consideradas en este trabajo y que, a nuestro entender, pueden ser abordadas como contenido transversal de diferentes materias. Esta transversalidad exige un esfuerzo mayor de coordinación entre los formadores universitarios. También abogamos por la transversalidad para el trabajo en los centros de enseñanza no universitaria con alumnos de diversas lenguas y procedencias. Huguet et al. (2011) recomiendan la búsqueda de consenso entre los profesionales y la coordinación entre el profesorado adoptando una perspectiva comunicativa de la adquisición de L2.

La normativa educativa estatal y gallega respecto al alumnado extranjero, desde el principio de atención a la diversidad, destaca la necesaria atención al desarrollo de sus competencias comunicativas y diversas investigaciones en nuestro contexto. Por ejemplo, Santos Rego, Godás y Lorenzo (2013) y Santos Rego, Priegue y Crespo (2017) subrayan la importancia de atender de forma especial la competencia lingüística en alumnos de origen inmigrante por su relación con el rendimiento académico general. A la luz de los resultados y también como una implicación más en relación con la formación inicial del profesorado, estimamos fundamental trabajar a fondo para que los estudiantes de maestro de Educación Infantil adquieran un buen estilo comunicativo que facilite el desarrollo adecuado de los niños y niñas con especial atención a aquellos que, como muchos inmigrantes, proceden de culturas diferentes a la del alumnado autóctono, de forma que se sientan comprendidos, acogidos y puedan establecer sólidos lazos afectivos en el ámbito del aula que faciliten el intercambio social, el aprendizaje lingüístico y su desarrollo integral como personas.

La importancia de las cuestiones abordadas (no olvidemos que respecto al ámbito lingüístico numerosas investigaciones destacan la importancia de la lengua vehicular de aprendizaje en relación con el nivel de éxito escolar) nos invitan a profundizar en nuestro estudio, pero dejamos para una ampliación del mismo otros aspectos sin duda interesantes como, por ejemplo, las diferencias entre los centros formadores respecto a las variables consideradas y la presentación de otros análisis estadísticos que arrojan más luz sobre los datos recogidos y su relación con otros resultados. Señalemos por otra parte que, como formadores de futuros docentes de Educación Infantil, estamos comprometidos en la actualidad con la introducción de cambios que propicien una mejora en la línea de lo apuntado anteriormente puesto que esta aportación forma parte de un estudio amplio que pretende ligar investigación, acción y formación. Por ejemplo, en materias del área de Didáctica y Organización Escolar usamos estrategias como la enseñanza con casos, el trabajo por proyectos, el trabajo cooperativo y los análisis autobiográficos introduciendo elementos que permitan analizar situaciones recogidas en la realidad de las aulas y de los centros que permitan desarrollar competencias en una línea de inclusión y educación intercultural. Con esta orientación creemos responder a las recomendaciones de la Unión Europea recogidas en diversos informes, el más reciente el Informe de la Educación y la Formación de 2019 recientemente presentado en la 2a Cumbre de Educación. No hemos de olvidar que en todo el mundo, según los datos recogidos en los dos últimos Informes de la OCDE (OCDE/EU 2018 y OCDE, 2018) los migrantes, como resume Sánchez-Montijano (2019), "tienden a tener peores niveles de integración educativa, económica y social que los nacidos 
en el país de acogida” (p. 249). En el caso de niños de edad inferior a seis años el acceso a una Educación Infantil de calidad, con profesorado competente para abordar retos como los que hemos citado en este trabajo, resulta crucial para elevar su nivel educativo y promover la cohesión social favorecedora del desarrollo y prosperidad del país de acogida.

\section{REFERENCIAS}

ACNUR (2019). Informe Anual 2018. Madrid: Agencia de la ONU para los Refugiados. Comité Español.

Aguilar-Idáñez, M. J. y Buraschi, D. (2017). Migrantes y refugiados: apuntes clave para un nuevo relato. Revista Lusófona de Educação, 37(37), 103-116. DOI: https://doi.org/10.24140/ issn.1645-7250.rle37.07

Baráibar, J. M. (2005). Inmigración, familias y escuela en Educación Infantil. Madrid: Los Libros de la Catarata/CIDE-MEC.

Chireac, S. M., Serrat, E. y Huguet, A. (2011). Transferencia en la adquisición de segundas lenguas. Un estudio con alumnado rumano en un contexto bilingüe. Revista de Psicodidáctica, 16(2), 267-289.

Cohen, L. y Manion, L. (1990). Métodos de investigación educativa. Madrid: La Muralla.

Comisión Europea (2019). Education and Training. Monitor 2019. Luxemburgo: Oficina de Publicaciones de la Unión Europea. Recuperado de https://bit.ly/2oXVisG

Consejo de la Unión Europea (2014). Conclusiones del Consejo de 20 de mayo de 2014 sobre la formación efectiva de los docentes. 2014 / C 183/05. Recuperado de https://bit.ly/2p3D35b

Cummins, J. (2000). Lenguaje, poder y pedagogía. Niños y niñas bilingües entre dos fuegos. Madrid: Morata.

Faria, L. (2008). Motivação para a Competência. Porto: Livpsic.

Fuentes Abeledo, E. (2009). Formación de maestros y Practicum en el contexto de cambio curricular desde la perspectiva de la convergencia europea. En M. Raposo, E. Martínez, et al. (Coord.), El Practicum más allá del empleo (pp. 103-124). Santiago de Compostela: Servicio Publicaciones de la Universidad de Santiago de Compostela.

Huguet, A. et al. (2011). Inmigración, lengua y escuela en sociedades bilingües. Revisión de estudios. Revista Interuniversitaria de Formación del profesorado. 72(25,3), 137-159.

Ibarra, J. (2012). Integración lingüística del alumnado inmigrado. Textos de didáctica de la lengua y la literatura, (61), 9-21.

Lillvist, A., Sandberg, A., Sheridan, S. y Williams, P. (2014). Preschool teacher competence viewed from the perspective of students in early childhood teacher education. Journal of Education for Teaching, 40(1), 3-19. DOI: https://doi.org/10.1080/02607476.2013.864014

Lessenich, S. (2019). La sociedad de la externalización. Barcelona: Herder.

Lomas, C. y Ruiz, U. (2011). Competencia comunicativa y educación democrática. Textos, (58), 5-8.

López, M., Martín, E., Montero, N. y Echeíta, G. (2013). Concepciones psicopedagógicas sobre los procesos de inclusión educativa: variables que las modulan y perfiles que las agrupan. Infancia y Aprendizaje, 36(4), 455-472. DOI: https://doi.org/10.1174/021037013808200285 
McMillan, J. y Schumacher, S. (2010). Research in Education: Evidence-Based Inquiry. Harlow: Pearson Addison Wesley.

Ministerio de Educación y Ciencia (MEC) (2006). Ley Orgánica 2/2006, de 3 de mayo, de Educación. BOE, 106, pp. 17158-17201. Recuperado de http://www.boe.es/boe/dias/2006/05/04/ pdfs/A17158-17207.pdf

Ministerio de Educación y Ciencia (MEC) (2007). Orden ECI/3854, por la que se establecen los requisitos para la verificación de los títulos universitarios oficiales que habilitan para el ejercicio de la profesión de Maestro en Educación Infantil. BOE, 312, pp. 53735-53738. Recuperado de http://www.boe.es/boe/dias/2007/12/29/pdfs/A53735-53738.pdf

Moreno, C. (2006). La enseñanza de español como lengua extranjera en contexto escolar. Madrid: Libros de la Catarata.

Navarro, J. L., Huguet, A. y Sansó, C. (2014). Competencias lingüísticas y alumnado inmigrante en Cataluña. El caso del colectivo hispanohablante. Educación Siglo XXI, 17(2), 361-382. DOI: https://doi.org/10.5944/educxx1.17.2.11495

Novick, N., Cauce, A., y Grove, K. (1996). Competence self-concept. En B. Bracken (Ed.), Handbook of self-concept: Development, social and clinic considerations (pp. 210-258). New York: John Wiley \& Sons.

Nurosis, M. (1993). SPSS. Statistical data analysis. New York: SPSS Inc.

Ribas, C., Iglesias, G., Siqués, C. y Vila, I. (2006). Materiales y actividades para las aulas de acogida. Barcelona: Horsori.

Sánchez Montijano, E. (2019). Objetivo 16. La cohesión social sobre la base de la integración. En L. Cachón, L. y M. Aysa-Lastra (Eds.), El Pacto Mundial para la emigración segura, ordenada y regular. Un modelo para armar (pp. 248-252). Barcelona: Hacer.

Santos Rego, M. A., Godás, A. y Lorenzo, M. (2013). Rendimiento académico y diversidad cultural: el eje lingüístico. Revista Española de Pedagogía, 71(256), 461-478.

Santos Rego, M. A., Priegue D. y Crespo, J. (2017). Aprendizaje escolar y percepción de competencia lingüística en alumnos de origen inmigrante. Revista Lusófona de Educação, (37), 181-196.

Siguán, M. (1998). La escuela y los inmigrantes. Barcelona: Paidós.

Vasseur, M. T. (2006). La cultura en la enseñanza y el aprendizaje de las lenguas. Confrontaciones, articulaciones, choques. Cuestiones para la formación de los docentes en contextos multilingües. Textos de didáctica de la lengua y la literatura, (42), 13-29.

Vila, I. (2007). Lenguas. En X. Besalú, Educar en sociedades pluriculturales (pp. 15-175). Barcelona: Wolters Kluwer. 